\title{
Aprendizaje colaborativo en tiempos de pandemia hacia la construcción de un eje socioeducativo
}

\section{Collaborative learning in times of pandemic towards the construction of a socio-educational axis}

\author{
Luis Guanipa \\ Iguanipa1@uc.edu.ve \\ https://orcid.org/0000-0003-4686-945X \\ Ginoid N. Franco \\ gfranco@uc.edu.ve \\ https://orcid.org/0000-0002-5410-9381
}

Universidad de Carabobo (UC). Facultad de Ciencias de la Educación, Venezuela.

Recibido: $18 / 08 / 21$

Aceptado: 30/10/21

\section{Resumen}

En la actualidad se ha desarrollado la Educación a Distancia gracias a la existencia de las Tecnologías de Información y Comunicación. Es por ello, que el propósito de esta investigación fue consolidar un eje socioeducativo basado en la educación a distancia que coadyuve al aprendizaje colaborativo en el área de Estudios de Postgrado de la FaCE-UC en tiempos de pandemia, sustentado con aprendizaje colaborativo, significativo, constructivismo, enseñanza sincrónica y asincrónica. Por otra parte, el método fue la hermenéutica de Gadamer. Se llegó a la conclusión, que tanto los docentes como los estudiantes expresaron que, mediante las herramientas de comunicación digital, plataformas y redes sociales se pudo adquirir el conocimiento de manera adecuada, mediante procesos sincrónicos y asincrónicos, además consideran que esto ayudará a cambiar la cultura presencial abriendo paso la construcción de una cultura bimodal y virtual.

Palabras clave: Educación a distancia, aprendizaje, constructivismo.

\section{Abstract}

At present, Distance Education has been developed thanks to the existence of Information and Communication Technologies. That is why the purpose of this research was to consolidate a socio-educational axis based on distance education that contributes to collaborative learning in the area of Postgraduate Studies of the FaCE-UC in times of pandemic, supported by collaborative, meaningful learning, constructivism, synchronous 
and asynchronous teaching. On the other hand, the method was Gadamer's hermeneutics. It was concluded that both teachers and students expressed that through digital communication tools, platforms and social networks it was possible to acquire knowledge in an appropriate way, through synchronous and asynchronous processes, they also consider that this will help to change the culture face-to-face, paving the way for the construction of a bimodal and virtual culture.

Key Words: Distance education, learning, constructivism.

\section{Introducción}

\section{Devenir de la Inserción Tecnológica en la Educación Universitaria a nivel internacional}

Los procesos comunicativos han evolucionado desde el lenguaje articulado, hasta la apertura a la era de la información y comunicación, la cual generó modificación de varios contextos, incluyendo el educativo, ya que la red pública internet trajo consigo que los procesos de búsqueda, mecanismos y procedimientos de información sean más rápidos; sin embargo, hoy día lo que se busca es ir más allá mediante una "Gestión de Conocimiento" donde se generen reflexiones de la información y se cumpla con el intercambio desnacionalizado del conocimiento (Guanipa, 2019).

Dentro de este marco de perenne transformación, las Tecnologías de Información y Comunicación (TIC) no fueron creadas inicialmente para el desarrollo educativo; sino el desarrollo económico, pero es innegable que paralelo a su desarrollo surgió oportunamente un nuevo paradigma de la enseñanza y del aprendizaje, partiendo de la utilización de las TIC como reforzamiento a la educación presencial, luego la educación mixta (presencial y virtual) favoreciendo la flexibilidad que algunos sistemas educativos formales necesitaban y convirtiéndose así en una propuesta importante de innovación e inclusión hasta llegar a la educación a distancia, en ésta última como plantea Malagón, Rodríguez y Ñanez (2019) se ha convertido en punto de referencia al momento de estructurar programas o universidades con este enfoque "La Universidad Nacional de Educación a Distancia (UNED) en España, la Fernuniversität Hagen en Alemania, la Open University en Reino Unido, la Universidade Aberta en Portugal, entre otras a nivel europeo" (p. 26).

Por otra parte, también existe experiencia en los Estados Unidos donde se ha presentado un notable crecimiento en el número de instituciones educativas privadas y estatales que ofertan programas de pregrado, postgrado y educación continua, destacando las Universidades de Berkeley, Michigan y Florida, en el ámbito privado la universidad de Harvard, Western Governors University (WGU) y Phoenix University, la cual se considera como la mayor universidad de educación a distancia del país (Malagon et al, 2019). 
Es conveniente resaltar en relación a la educación a distancia que modalidad de educación no determina en sí misma un cuerpo único de saberes, metodologías, procedimientos; sino, que recoge en su hacer particularidades propias del contexto en los que se desenvuelve, tal como resalta Villalonga (2015) al conceptualizarla como:

El uso de técnicas pedagógicas, recursos y medios de comunicación específicos para facilitar el aprendizaje y la enseñanza entre alumnos y docentes que están separados por el tiempo o la distancia. Las técnicas, recursos y medios de comunicación dependen de factores como: la asignatura, las necesidades y el contexto del alumno, la competencia y experiencia del docente, los objetivos de la instrucción, las tecnologías disponibles y la capacidad institucional (p. 5).

En este sentido, debido al éxito de esta modalidad en educación se ha impulsado su inserción en la mayoría de las universidades situadas en países desarrollados ya que se articulan la tecnología y los procesos formativos, mientras que en los países en vías de desarrollo existen universidades transitando el camino de la actualización de recursos tecnológicos, humanos, económicos y de infraestructura. Tal es el caso, de universidades en México, Costa Rica, Colombia, El Salvador, Paraguay, Ecuador, Panamá, Argentina y Puerto Rico que han aprobado marcos legales que autorizan a las universidades en la administración de los programas de pregrado y postgrado cien por ciento virtuales.

Esta visión del mundo ha permeado en Venezuela donde, existen universidades públicas que trabajan completamente a distancia dentro de las que se encuentra: la Universidad Nacional Abierta (UNA) como la primera del país en utilizar sistemas no tradicionales, tales como: educación a distancia, tele educación, enseñanza programada, entre otros; fue concebida para operar a escala nacional y posee actualmente más de sesenta y siete (67) sedes extendidas a lo largo de todo el país, convirtiéndola en una de las universidades con más sedes en la Nación. Seguidamente; aquellas bajo las modalidades mixtas la Escuela de Estudios Táctico Navales (ETNAV), Universidad del Zulia (LUZ) y dentro de las privadas: Universidad Rafael Belloso Chacín (URBE), Universidad Yacambú (UNY), Universidad Católica Andrés Bello (UCAB) y Universidad Católica Cecilio Acosta (UNICA).

Dentro de las Universidades venezolanas públicas con trayectoria académica se encuentra la Universidad de Carabobo, la cual desde el año 2007 realizó proyectos pilotos para el uso adecuado de las tecnologías en ambientes educativos reales, con el fin de fortalecer el sistema educativo, difundir conocimientos, brindar acceso a la información, promover un aprendizaje de calidad y efectivo, y prestar servicios de forma más eficaz donde las clases de Pregrado son presenciales y en el Área de Estudios de Postgrado de las diferentes Facultades gran parte de los programas son bajo administración bimodal (Mixtas). Ahora bien, en la Facultad de Ciencias de la Educación (FaCE) de la precitada institución, se posee la experiencia en entornos virtuales donde emergen evidencias a nivel de pregrado y postgrado de integración curricular de las TIC en la educación, así como formación a los profesores para lograr su implantación en el Aprendizaje colaborativo en tiempos de pandemia hacia la construcción de un eje socioeducativo. - Eduweb, 2021,
septiembre-diciembre, v.15, n.3./48-61 
currículo: Herramientas Digitales para Educadores, Estrategias apoyadas en el uso de las TIC, entre otros.

Por lo que, en la actualidad su inserción en la Universidad de Carabobo ha encontrado sustento legal en el ámbito nacional e interno específicamente con la reciente aprobación de la Normativa de Educación a Distancia (2019) la cual en su artículo 2 la consagra como aquella de carácter "formativo, que utilice recursos de la plataforma tecnológica educativa de la institución y que se adapte a una de las modalidades de ejecución, utilizando para ello las Tecnologías de Información y Comunicación (TIC) en la gestión docente" (p. 10).

Es así como, en los estudios de postgrado de la FaCE en los distintos programas se desarrollan procesos educativos virtuales en respuesta a las exigencias de los escenarios sociales y el contexto educativo internacional que demanda la sociedad del siglo XXI, siendo importante las habilidades y destrezas en el manejo de recursos tecnológicos.

\section{Educación a Distancia a la luz del aprendizaje colaborativo en tiempos de pandemia.}

El contexto actual mundial de desarrollo de la pandemia covid-19 ha obligado a las Instituciones de Educación Superior (IES) a reestructurar los procesos académicos para asegurar la prosecución académica a distancia, así como articular aspectos institucionales y de la formación para la investigación y producción de conocimientos de los estudiantes. Por ello, en razón a la suspensión de actividades académicas presenciales, la FaCE se adapta a las nuevas realidades y exigencias de los tiempos, cónsona con su sagrada misión de formar profesionales de la docencia de alta calidad académica que respondan a las exigencias del país y el mundo en sus distintos momentos históricos; de allí que, en los estudios de postgrado se asume la administración de la modalidad virtual así como la adecuación curricular de manera excepcional en sujeción al Decreto Estado de Excepción de Alarma $N^{\circ} 4.160$, de fecha $13 / 03 / 2020$ y prorrogado mediante Decreto № 4.186 de fecha 12/04/2020, ante la pandemia Covid-19.

En este sentido, se hace presente la necesidad de repensar la administración curricular de los programas de los estudios de postgrado en la Facultad de Ciencias de la Educación de la Universidad de Carabobo (UC) y en el caso de la Maestría en Investigación Educativa, objeto de estudio; se ha asumido el desarrollo de las unidades curriculares de forma abierta y flexible para dar respuesta institucional a la situación de contingencia pandémica sobrevenida y los inconvenientes en la conectividad intermitente, en aras de asegurar la calidad, inclusión, construcción y transferencia del conocimiento de manera efectiva y productiva en estos entornos virtuales. 
Es por ello, que la direccionalidad y alcance de la investigación se sitúa en el cuatrimestre Mayo-Julio con la atención de los participantes en el mencionado programa en observancia a reducir la brecha de inequidad en la medida de las posibilidades de estudiantes y facilitadores impulsando la autogestión de aprendizaje o aprendizaje autónomo relacionados no sólo con los contenidos de aprendizaje sino también con los intereses particulares de los participantes; de allí el seguimiento permanente y comunicación para valorar la calidad de esta experiencia en cuanto a la administración curricular a distancia, modalidad que en futuro cercano, será necesaria y pertinente. Todo ello conduce a los autores a la siguiente pregunta de investigación: ¿cómo se puede desarrollar la Educación a Distancia a la luz del aprendizaje colaborativo en tiempos de pandemia? y los subsiguientes propósitos de investigación

\section{Propósito General}

Construir un eje socioeducativo basado en la educación a distancia a la luz del aprendizaje colaborativo en tiempos de pandemia.

\section{Propósitos Específicos}

- Comprender la realidad socioeducativa de la educación a distancia en el contexto investigativo universitario.

- Interpretar las diferentes miradas de la educación a distancia en el área de Estudios de Postgrado de la FaCE-UC.

- Consolidar un eje socioeducativo basado en la educación a distancia que coadyuve al aprendizaje colaborativo en el área de Estudios de Postgrado de la FaCE-UC en tiempos de pandemia.

\section{Relevancia del Estudio}

Los resultados esperados de esta indagación, servirán de aporte a los esfuerzos que viene realizando la FaCE-UC en la ejecución de acciones bajo el uso de herramientas tecnológicas que posibiliten el logro de la prosecución académica en postgrado. De igual forma, contribuirá significativamente a brindar aportes importantes de gran relevancia académica- curricular, que se constituyan como antecedente en nuestra Facultad y Universidad para abrir horizontes en esta modalidad a virtual, garantizando el derecho a la educación en el bien entendido de que estos procesos deben ocurrir al amparo de criterios de calidad académica y compromiso institucional.

Por otra parte, superar esquemas tradicionales en el contexto de comprender el caos del mundo hacia cierta unidad, para vislumbrar que, en una sociedad de constantes e incesantes cambios, si se tiene un sustento legal y constitucional en el empleo de las TIC en la educación, resulta imperante abrirse a nuevas experiencias y las diversas las percepciones de docentes y participantes. De manera que, esta experiencia analizada, estudiada y comprendida arrojará luces hacia la orientación en la transformación curricular. Finalmente, el aporte hacia las Ciencias de la Educación conllevará a la Aprendizaje colaborativo en tiempos de pandemia hacia la construcción de un eje socioeducativo. - Eduweb, 2021,
septiembre-diciembre, v.15, n.3./48-61 
๔eduweb

Revista de Tecnología de Información y Comunicación en Educación • Volumen 15, ํㅜ 3. Septiembre-diciembre 2021

sustentación epistémica y ontológica de las iniciativas institucionales que giran en torno a la incorporación de las TIC en el currículo y, por ende, en su quehacer organizacional.

\section{Aprendizaje colaborativo, significativo y constructivismo}

El aprendizaje colaborativo se basa en supuestos epistemológicos diferentes y tienen su origen en el constructivismo social, el cual recoge la esencia los fundamentos filosóficos del aprendizaje colaborativo. El aprendizaje colaborativo se produce cuando los estudiantes y los profesores trabajan juntos para crear el saber; es decir, parte de la base de que las personas crean significados juntas y que el proceso las enriquece y las hace crecer.

En vez de dar por sentado que el saber existe en algún lugar de la realidad "exterior" y que espera ser descubierto mediante el esfuerzo humano, el aprendizaje colaborativo, en su definición más estricta, parte de la base de que el saber se produce socialmente por consenso entre compañeros versados en la cuestión, teniendo en cuenta, que el saber es algo que construyen las personas hablando entre ellas y poniéndose de acuerdo. Cabe destacar, que en el aprendizaje colaborativo se quiere evitar que los estudiantes se hagan dependientes del profesor como autoridad en los contenidos de la asignatura o en los procesos grupales; por lo tanto, no le corresponde al profesor la supervisión del aprendizaje en grupo, sino que su responsabilidad consiste en convertirse, junto con los estudiantes, en miembro de una comunidad que busque el saber. (Barkley, Cross y Howell, 2007)

Por otra parte, tenemos el aprendizaje significativo el cual surge cuando el estudiante como constructor de su propio conocimiento relaciona los conceptos a aprender y les da sentido a partir de la estructura conceptual que ya posee. El aprendizaje se construye al relacionar los conceptos nuevos con los conceptos que ya se poseen y otras al relacionar los conceptos nuevos con experiencias que ya se tienen.

Es por ello, que para el Aprendizaje Significativo se desarrolle debe cumplir con dos condiciones básicas "Disposición del sujeto a aprender significativamente y que el material a aprender sea potencialmente significativo, es decir relacionable con sus estructuras de conocimientos" (Román y Diez, 1990, p. 74). Para que se puedan dar las disposiciones mencionadas anteriormente, hay que buscar la forma de generar la necesaria motivación a partir de los intereses de los estudiantes y construir previamente el material de manera significativa, para hacer esto hay que saber en qué contexto se desenvuelve los individuos a los que se les va a impartir los conocimientos y tener dominio conceptual del tema que se va a impartir, para poder relacionar de manera significativa el aprendizaje.

No se puede soslayar, el Constructivismo cuyas bases, anteceden a la moderna psicología y se remonta al movimiento intelectual que surge en Grecia en el siglo V a. c., conocido como Sofística. Los sofistas revierten la concepción geocentrista, que hasta el 
momento había imperado, en una concepción antropocentrista, en la que el hombre, la sociedad y la educación, se revelan como importes y dignos de estudio.

Fuenmayor y Orellana (2002) plantean que Protágoras (480-410 a. c.) y Giorgias (380 a. c.), han sido considerados entre los principales representantes de tal movimiento intelectual, los cuales nos conducen hacia los planteamientos actuales del constructivismo radical. Es el hombre quien determina la existencia de las cosas, éstas son porque el hombre las conoce, si no las conoce no son, en palabras del constructivista radical, no hay realidad independiente del observador. En la actualidad, la postura constructivista se alimenta de diversas corrientes, el enfoque psicogenético piagetano, la teoría de los esquemas cognitivos, la teoría ausubeliana de la asimilación y el aprendizaje significativo, la psicología sociocultural vigotskiana, entre otras. Es por ello, que el proceso de construcción de los aprendizajes depende de dos aspectos fundamentales, de los conocimientos previos que tengan de la nueva información y de la actividad externa o interna que el aprendiz realice al respecto.

Por lo dicho anteriormente, hay que tener en cuenta que ésta teoría equipara al aprendizaje con la creación de significados a partir de Experiencias. Para ser exitoso y duradero, el aprendizaje debe incluir los tres factores cruciales siguientes: Actividad (ejercitación), Concepto (conocimiento) y cultura (contexto).

\section{Enseñanza sincrónica y asincrónica.}

La enseñanza sincrónica se imparte y se recibe al mismo tiempo; dicha enseñanza en aulas virtuales y mixtas transcurre en lo que se denomina "tiempo real". El tiempo real es cuando todos los participantes de una actividad se involucran en esa actividad al mismo tiempo, independientemente de su ubicación, en consecuencia, se necesita que los estudiantes asistan a una lección virtual durante la cual todos los participantes deben iniciar sesión al mismo tiempo, esa lección es una enseñanza sincrónica impartida en tiempo real (Cabero et al., 2004, pp. 30-31).

Por otra parte, se encuentra la enseñanza asincrónica la cual no depende del tiempo real, en este caso los estudiantes pueden realizar la interacción sin estar en tiempo real mediante una lección grabada para otro día, foro de discusión y realizar sus aportes ya que no es necesario que los participantes estén conectados al mismo tiempo para concluir sus asignaciones (Cabero et al., 2004, pp. 30-31). 


\section{Complexus pentateórico}

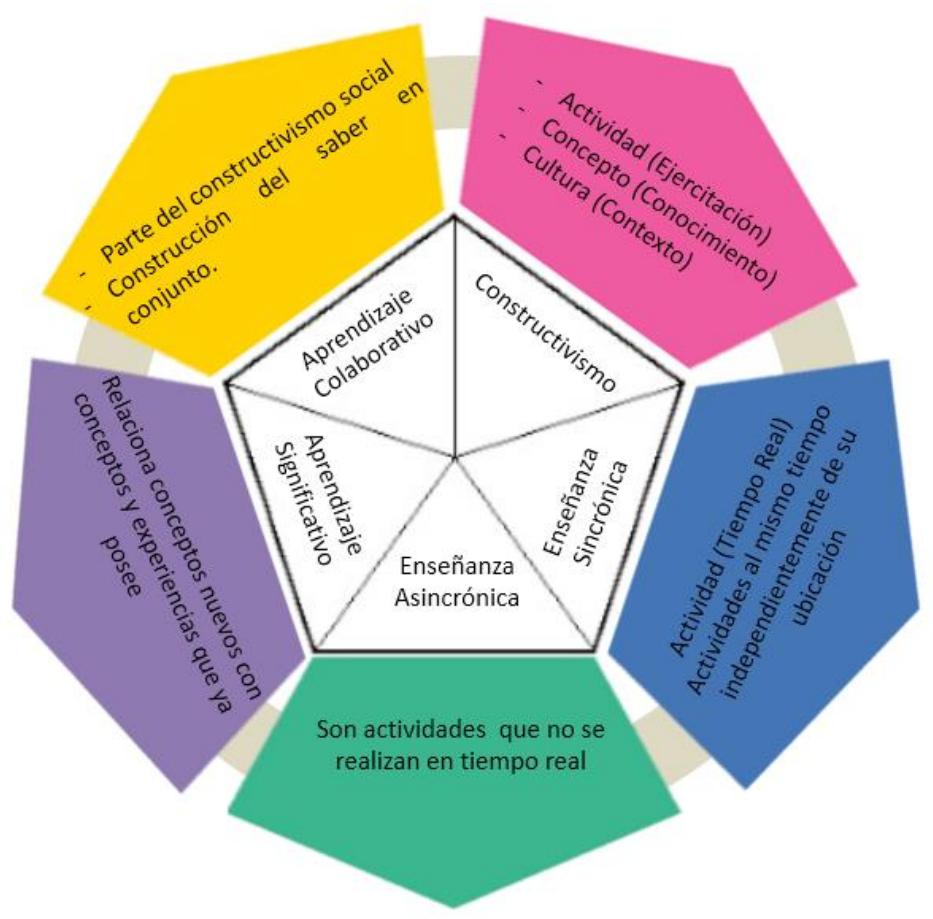

Fuente: Autores (2020) Infografía Pentateórica de la EaD.

Teniendo en cuenta que las características del aprendizaje colaborativo una es que tiene como origen el constructivismo social y la otra es la construcción del saber en conjunto, por otra parte se encuentran las características del constructivismo del cual surgen tres vertientes como son: actividad (ejercitación), concepto (conocimiento) y cultura (contexto), además el aprendizaje significativo tiene como característica la relación de los conceptos nuevos con los conceptos y experiencias que ya posee, no se puede soslayar la enseñanza sincrónica que es aquella que se realiza en "tiempo real" y enseñanza asincrónica que no es realizada en tiempo real.

En estas teorías ocurre un complexus ya que una de las características del constructivismo como se nombró anteriormente es el concepto (conocimiento), mientras en el aprendizaje colaborativo se busca la construcción del "saber" que en este caso también es conocimiento, pero para que se desarrolle ese conocimiento en el aprendizaje colaborativo al igual que en el constructivismo se debe realizar actividades teniendo en cuenta el contexto, lo único en que se diferencian es que en el aprendizaje colaborativo el aprendizaje se desarrolla en conjunto; en cuanto al aprendizaje significativo se caracteriza por la relación los conceptos nuevos que es el conocimiento del constructivismo y el saber del aprendizaje colaborativo con los conceptos y experiencias que ya se poseen - que no es más que el contexto del constructivismo - se puede entonces evidenciar la recursividad de estas teorías y que a su vez se relacionan a la 
hora de impartir EaD de manera sincrónica y asincrónica mediante herramientas de comunicación digital, plataformas y redes sociales, donde se puede decir que este complexus pentateórico ocurre de manera compleja mediante principios hologramáticos, dialógicos y recursivos.

\section{Método}

Dentro del Método se encuentra el "Círculo Hermenéutico" que explica como "el movimiento de la comprensión que va del todo a la parte y de la parte al todo" (Gadamer, 1998, p. 63). En el que teje el discurso a partir de la comprensión analítica. Aclara el autor en su exposición: "El análisis comprensivo de las fuentes y su interconexión con las localidades problemáticas permitirá hacer congruente el hilo discursivo, en el razonamiento interpretativo y de aplicación de los contenidos a obtenerse" (Gadamer, 1993, p. 193).

La interpretación funde lo aspirado por el intérprete y lo ofrecido significativamente por el texto o el acto humano. En este sentido, alude a una suerte de condición ubicua del intérprete sobre el interlocutor, es decir, coloca en la subjetividad razonablemente crítica del intérprete, la responsabilidad de la interpretación, cuando aclara que el trabajo de éste no es simplemente reproducir lo que dice en realidad el interlocutor, sino que tiene que hacer valer su opinión de la manera que le parezca necesaria, teniendo en cuenta la autenticidad de la situación dialógica en que sólo él se encuentra como conocedor del lenguaje de las dos partes. En cuanto a la Aplicación, Gadamer la considera como un momento del proceso hermenéutico tan esencial e integral como la comprensión y la interpretación; es por ello, que la facticidad es el reflejo de validación de los enunciados interpretados al dibujar nuevos horizontes desde lo ya construido hacia lo construible en una relación de apropiación discursiva entre el hermeneuta y el objeto del conocimiento. (Gadamer, 1993, p.p. 193-194)

Esta preocupación interesa a Gadamer, para quien la aplicación no quiere decir aplicación ulterior de una generalidad dada, comprendida primero en sí misma a un caso concreto; ella es más bien la primera verdadera comprensión de la generalidad que cada texto dado viene a ser para nosotros: La comprensión es una forma de efecto, y se sabe a sí misma como efectual. Como forma de efecto, la aplicación, en tanto comprensión de una generalidad, como horizonte último, y luego de comprendido el soporte teórico e interpretado sobre la facticidad del mundo de la vida, devendrá la construcción teórica. Cabe destacar, que en la investigación se realizó una triangulación del corpus (textos y teorías), de los sujetos abordados (Docentes y participantes de la maestría Investigación Educativa) y la reflexión de los investigadores. De esa triangulación surgieron en relación a la EaD, las siguientes categorías: Aprendizaje, Enseñanza, Redes Sociales, Herramientas de comunicación digital, plataformas, categorías que son los cimientos de la investigación. 


\section{Matrices Categoriales}

Cabe destacar, que para el desarrollo de las matrices categoriales se desarrolló una codificación axial que "es comenzar el proceso de reagrupar los datos que se fracturaron durante la codificación abierta. En la codificación axial, las categorías se relacionan con sus subcategorías para formar unas explicaciones más precisas y completas sobre los fenómenos" (Strauss y Corbin, 2002, p. 135). Es por ello, que se desarrollan de la siguiente manera (Categorías, subcategoría, Axiomas Subcategoriales y Relaciones categoriales), como se mencionó anteriormente las categorías "son conceptos derivados de los datos, que representan fenómenos y que son ideas analíticas pertinentes que emergen de nuestros datos" (Strauss y Corbin, 2002, p.124), estas categorías surgieron después de haber hecho un detallado análisis, línea por línea del corpus (textos abordados) y los sujetos entrevistados, de allí surgieron subcategorías que "son aquellas que responden preguntas sobre los fenómenos tales como cuándo, dónde, por qué, quién, cómo y con qué consecuencias, dando así a los conceptos un mayor poder explicativo" (Strauss y Corbin, 2002, p. 136).

Una vez realizado las categorías y subcategorías se incorpora los axiomas subcategoriales teniendo en cuenta que un axioma es "un supuesto no demostrado (ni demostrable), cuya función consiste en permitir la demostración de otras fórmulas de la teoría" (Martínez, 2004, p. 243). Se puede decir entonces que es una proposición asumida dentro de un cuerpo teórico sobre la cual descansan otros razonamientos y proposiciones deducidas de esas premisas. Luego de haberse desarrollado los axiomas subcategoriales se plasmaron las categorías que se relacionan entre sí para luego desarrollar esa relación en el aporte al conocimiento.

Tabla 1.

Matriz Categorial

\begin{tabular}{|c|c|c|c|}
\hline \multirow[t]{3}{*}{ Aprendizaje } & Colaborativo & $\begin{array}{l}\text { Origen en el constructivismo social, } \\
\text { construcción del saber en conjunto }\end{array}$ & \multirow{8}{*}{$\begin{array}{c}\text { Aprendizaje, } \\
\text { enseñanza, } \\
\text { redes sociales, } \\
\text { herramientas } \\
\text { de } \\
\text { comunicación } \\
\text { digital y } \\
\text { plataformas. }\end{array}$} \\
\hline & Significativo & $\begin{array}{l}\text { Relación de conceptos nuevos con } \\
\text { conceptos y experiencias que ya se } \\
\text { poseen. }\end{array}$ & \\
\hline & Constructivista & $\begin{array}{l}\text { Actividad (ejercitación), concepto } \\
\text { (conocimiento) y cultura (contexto). }\end{array}$ & \\
\hline \multirow[t]{2}{*}{ Enseñanza } & Sincrónico & $\begin{array}{l}\text { Más social, tiempo real, conectividad } \\
\text { grupal. }\end{array}$ & \\
\hline & Asincrónico & $\begin{array}{l}\text { Actividades que no se realizan en } \\
\text { tiempo real. }\end{array}$ & \\
\hline \multirow[t]{3}{*}{ Redes Sociales } & WhatsApp & Foro chat, videos, tutoriales. & \\
\hline & Hangouts & $\begin{array}{l}\text { Video conferencia, presentaciones, } \\
\text { video foro. }\end{array}$ & \\
\hline & Zoom & $\begin{array}{l}\text { Video conferencia, presentaciones, } \\
\text { video foro. }\end{array}$ & \\
\hline
\end{tabular}




\begin{tabular}{|c|c|c|}
\hline & Google Meet & $\begin{array}{l}\text { Video conferencia, presentaciones, } \\
\text { video foro. }\end{array}$ \\
\hline \multirow{2}{*}{$\begin{array}{l}\text { Herramientas de } \\
\text { comunicación } \\
\text { digital }\end{array}$} & Blog & Enlaces, videos, comentarios. \\
\hline & Educaplay & Test, mapas, juegos, autoevaluación. \\
\hline \multirow[t]{3}{*}{ Plataformas } & Moodle & $\begin{array}{l}\text { Videos, materiales, asignaciones, } \\
\text { foros, tareas, evaluaciones. }\end{array}$ \\
\hline & Youtube & Videos tutoriales, canales educativos. \\
\hline & Classroom & $\begin{array}{l}\text { Materiales, videos, tareas, tutoriales, } \\
\text { comentarios, evaluaciones. }\end{array}$ \\
\hline
\end{tabular}

\section{Aporte al Conocimiento}

Una vez realizada la triangulación del corpus, sujetos abordados y la reflexión de los investigadores surgió este aporte al conocimiento dado por un complexus pentateórico por un lado y por otro el abordaje socioeducativo de los docentes y participantes de la maestría de Investigación Educativa de la FaCE-UC mediante herramientas digitales, plataformas y redes sociales como se muestra en la siguiente infografía:

EaD a la luz de la Realidad Socioeducativa en la Maestría de Investigación Educativa FaCE - UC

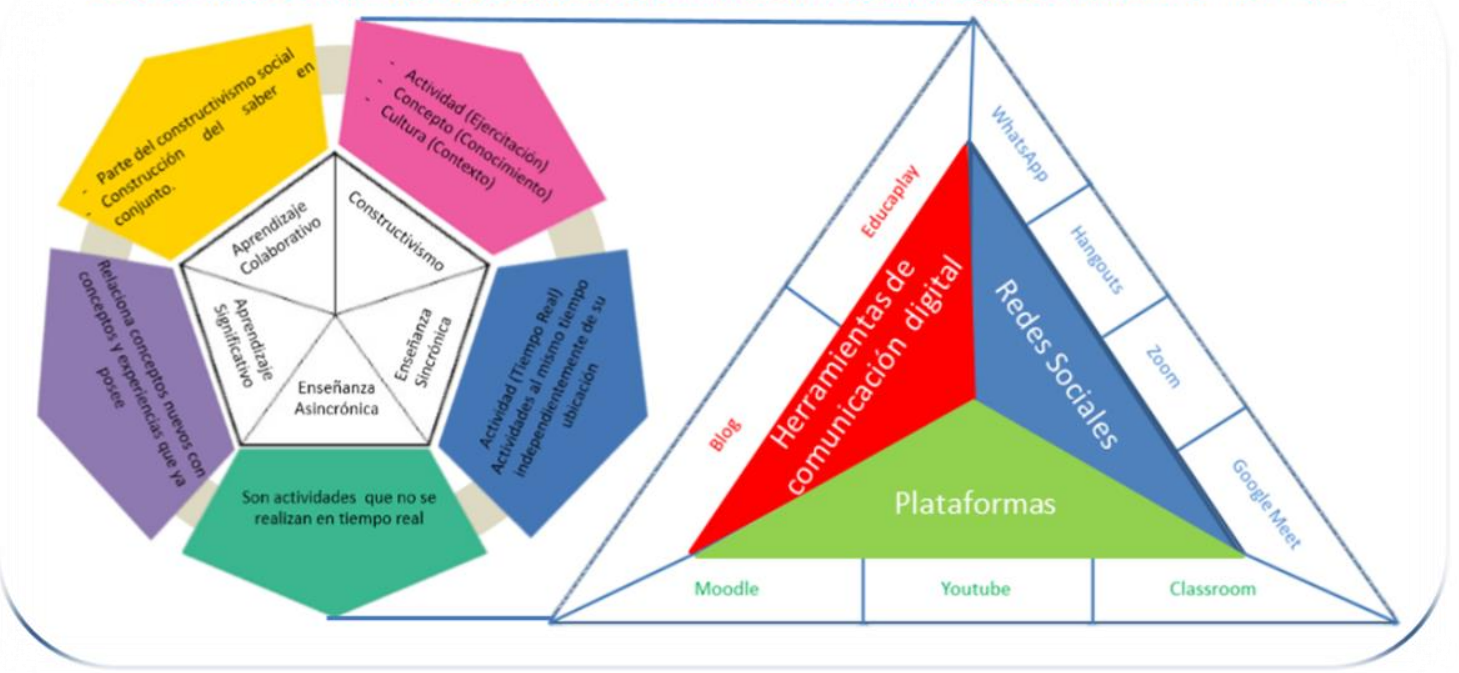

Fuente: Autores (2020) Infografía EaD a la luz de la Realidad Socioeducativa.

En el lado izquierdo de la Infografía EaD a la luz de la Realidad Socioeducativa (2020) se puede visualizar como el constructivismo, aprendizaje colaborativo y significativo hay puntos de encuentro como son el conocimiento, concepto o saber cómo se explicó en líneas anteriores, además hay también relación en cuanto a la cultura, contexto o experiencia y no se puede soslayar las actividades o ejercitación que a su vez se relacionan a la hora de impartir EaD de manera sincrónica (tiempo real) y asincrónica (cualquier momento que se acuerde) mediante herramientas de comunicación digital, plataformas y redes sociales que se encuentran del lado derecho del infograma, se 
puede decir entonces que ocurre complexus de manera compleja mediante principios hologramáticos, dialógicos y recursivos.

Cabe destacar, que en la Facultad de Ciencias de la Educación de la Universidad de Carabobo específicamente en la Maestría de Investigación Educativa normalmente el abordaje académico es Bimodal; es decir, con encuentro presencial y el otro es virtual en la semana de actividades académicas, donde a los participantes se les asigna actividades para ser desarrolladas generalmente desde la plataforma Moodle o en su defecto por classroom, mediante materiales digitales en (PDF, Videos, presentaciones, textos, audios, foros, tareas y evaluaciones); sin embargo, cuando comienza el aislamiento social por causa de la pandemia del COVID-19 fue necesaria la implementación total de herramientas para el desarrollo de las clases a distancia que serían desde ese momento cien por ciento virtuales, dentro de esas estrategias surgen de acuerdo a la realidad socioeducativa que se estaba manejando, herramientas digitales que dominaban los docentes y dependiendo de la conectividad que tenían los docentes y participantes los procesos fueron generalmente asincrónicos y muy pocas veces sincrónicos, ya que en muchas oportunidades no se contaba con el suministro eléctrico o no todos contaban con conectividad permanente en sus hogares y los cyber - locales donde prestan servicio de internet - se encontraban cerrados a causa del aislamiento social por la pandemia.

Por otra parte, en el lado derecho de la infografía se puede observar las plataformas que fueron empleadas como fueron Moodle, classroom, Google Meet y YouTube, para el desarrollo de las actividades (Materiales, videos, tareas, tutoriales, comentarios, evaluaciones) y en el caso de YouTube los docentes realizaron tutoriales que luego subieron a sus canales para que los participantes pudieran tener acceso a ellos mediante el Classroom, blog o WhatsApp para poder ampliar los canales de comunicación entre el docente y los estudiantes, la ventaja de estas herramientas es que se realizaban de manera asincrónica.

En cuanto a las redes sociales, una de las más utilizadas fue WhatsApp, donde el proceso de comunicación fluyó de manera más sencilla ya que mediante grupos, el docente podía comunicarle información, realizar foro chat y mandar videos o tutoriales para ayudar con el proceso educativo, la ventaja en cuanto a esta herramienta es que generalmente se utiliza de manera asincrónica; por otra parte, se manejaron redes sociales como Hangouts, Zoom y Google Meet para desarrollar (video conferencias, presentaciones, video foro).

También se realizaron abordajes de herramientas de comunicación digital mediante Blog donde se colgaban (enlaces, videos y comentarios), la otra herramienta fue Educaplay utilizada por docentes para desarrollar (Test, mapas, juegos con su autoevaluación). Es importante destacar que tanto los docentes como los estudiantes expresaron que mediante las herramientas de comunicación digital, plataformas y redes sociales se pudo adquirir el conocimiento de manera adecuada, pese en algunos casos la falta de 
electricidad y conectividad, consideran que esto ayudará a cambiar la cultura presencial, que tanto los docentes como participantes no tengan que tener gastos de traslado a las instituciones y como plantea la UNESCO "Es preciso aprovechar las Tecnologías de Información y Comunicación (TIC) para reforzar los sistemas educativos, la difusión de conocimientos, el acceso a la información, el aprendizaje efectivo y de calidad, y una prestación más eficaz del servicio" (UNESCO, 2015, p. 7).

En consecuencia, no basta con disponer de los más modernos medios, ellos no actúan solos. Al respecto, Facundo (2005) expresa que se requiere crear una cultura que fomente el cambio en las mediaciones pedagógicas, la superación de la vieja concepción de "enseñanza por exposición y aprendizaje por recepción" y la implantación de nuevos paradigmas de aprendizaje para aprovechar las tecnologías digitales. Esto quiere decir, que de manera ineludible la planificación, ejecución y evaluación del acto didáctico deben experimentar modificaciones; pues las formas y maneras de hacer docencia no pueden permanecer iguales a lo largo del tiempo, más aún considerando las implicaciones, consecuencias y efectos de esta era digital y la presencia en los regímenes de estudios universitarios de la modalidad mixta y virtual.

\section{Referencias}

Barkley, E., Cross, P. y Howell, C. (2007) Técnicas de aprendizaje colaborativo. Madrid: Ediciones MORATA, S. L.

Cabero, J., Llorente, M. y Román, P. (2004) Las Herramientas de la comunicación en el "Aprendizaje Mezclado". Revista Pixel-Bit. Revista de Medios y Educación, $\mathrm{N}^{\circ} \quad 023, \quad 27-41$. https://idus.us.es/bitstream/handle/11441/22780/09e4150d0ad1a010d5000000.p df? sequence $=1$ \&isAllowed $=y$

Facundo, A. (2005). Tecnologías de Información y Comunicación y Educación Superior Virtual en América Latina y el Caribe: Evolución, Características y Perspectivas. Bogotá: Los Libertadores/IESALC.

Fuenmayor, M. y Orellana, R. (2002). E Constructivismo en Educación. Barinas, Venezuela: Universidad Nacional Experimental de los Llanos Occidentales Ezequiel Zamora.

Gadamer, H. (1993) Verdad y Método. Salamanca: Ediciones Sigueme.

Gadamer, H. (1998) Verdad y Método II. Salamanca: Ediciones Sigueme.

Guanipa, L. (2019) Onto-epitemia de la realidad socioeducativa a la luz de la gestión del conocimiento en el contexto universitario venezolano. Área de Estudios de postgrado de la Universidad de Carabobo. Venezuela.

Malagón, L. Rodríguez, L. y Ñanez, J. (2019) Prácticas Pedagógicas y Educación a Distancia. Colombia: Universidad de Tolima.

Martínez, M. (2004) Ciencia y arte en la metodología cualitativa. México: Trillas.

Román, M. y Diez, E. (1990). Currículo y Aprendizaje. (2a.ed.). Madrid, España: Gráficas Monterreina, S.A. 


\section{Geduweb}

Revista de Tecnología de Información y Comunicación en Educación • Volumen 15, Nㅜ 3. Septiembre-diciembre 2021

Strauss, A. y Corbin, J. (2002) Bases de la investigación cualitativa. Técnicas y procedimientos para desarrollar la teoría fundamentada. Colombia: Editorial Universidad de Antioquía.

UNESCO (2015) Educación 2030 Declaración de Incheon Hacía una educación inclusiva y equitativa de calidad y un aprendizaje a lo largo de la vida para todos. Editorial: Organización de las Naciones Unidas para la Educación, la Ciencia y la Cultura.

Universidad de Carabobo (2019). Normativa de Educación a Distancia. Venezuela.

Villalonga, A. (2015) La educación superior a distancia. Modelos, retos y oportunidades. La Habana, Cuba: Oficina de la UNESCO. 